\title{
HACIA LA CONSTRUCCIÓN DE LA HISTORIA DE UN (DES) ENCUENTRO: LA RAZÓN FEMINISTA Y LA AGENCIA ANTIRACISTA Y DECOLONIAL EN ABYA YALA
}

\section{TOWARDS THE CONSTRUCTION OF HISTORY FROM A (DIS) AGREEMENT: FEMINIST REASON AND ANTIRACIST AND DECOLONIAL AGENCY IN ABYA YALA}

\author{
Yuderkys Espinosa Miñoso ${ }^{1}$ \\ Grupo GLEFAS \\ espinosayuderkys@gmail.com
}

Recibido: 1de mayo del 2017 / Aceptado: 28 de julio del 2017

Publicado: 7 de noviembre del 2017

Resumen: En este trabajo intento avanzar en la construcción de una genealogía de la relación entre la política feminista y las luchas antirracistas negras e indígenas en Abya Yala, desde una mirada decolonial. Se explora el surgimiento tardío de los movimientos y las luchas antirracistas, decoloniales y étnico-raciales en América Latina, observando la ideología del mestizaje y los procesos de occidentalización amplia como obstáculo por superar y enfrentar, incluso por el propio feminismo en su intento de descolonizarse y enfrentar la razón imperial racista instalada en sus simientes. Al final, analizo los costos que tiene para el feminismo en América Latina persistir en su mirada y tratamiento fragmentado de la opresión centrada en el género.

Palabras clave: antirracismo, feminismo decolonial, feminismo latinoamericano, opresión, género.

1 Dominicana, importante activista y productora de teoría con temas como feminismo decolonial, sexualidades y dinámicas racistas. Se desempeña como docente en varias universidades de América Latina. Co fundadora del grupo GLEFAS. Es autora de numerosos artículos y libros, entre los que se encuentra Escritos de una lesbiana oscura: reflexiones críticas sobre feminismo y política de identidad en América Latina (Ed. En la frontera, Buenos Aires). 
Summary: In this article, I attempt to move towards the construction of a genealogy about the relationship between feminist politics and antiracist black and indigenous struggles in Abya Yala, from a decolonial perspective. I explore the late rise of the antiracist, decolonial and ethnic-racial movements and fights in Latin America, observing the ideology of miscegenation and ample westernization processes as an obstacle to be overcome and faced, even by feminism, in its attempt to decolonize itself and face the racial imperial reason installed in its foundation. Finally, I analyze the cost for feminism in Latin America of persisting with this point of view and fragmented treatment of oppression centered on gender.

Keywords: antiracism, decolonial feminism, Latin American feminism, oppression, gender.

\section{Introducción}

En la introducción del libro Tejiendo de otro modo: Feminismo, epistemología y apuestas decoloniales en Abya Yala, que publiqué junto a Karina Ochoa y Diana Gómez (2014), nos hemos tomado algunas páginas para pensar la complicada relación entre el feminismo y las luchas indígenas y afrodescendientes en el lugar conocido, por su nombre colonial, como América Latina. A partir de un ejercicio de construcción de memoria recordamos cómo, desde finales de la década de los ochenta, se comienzan a perfilar luchas indígenas y afrodescendientes amplias, que interpelarán con voz cada vez más potente los Estados nación con su exigencia de autonomía (política, cultural, organizativa, epistemológica), y con su crítica al discurso euro centrado de las instituciones, la agenda internacional de derechos, el mundo del desarrollo y la política de los movimientos sociales urbanos locales e internacionales con su mirada universalista y sus ideales "de bien", centrados en la agencia individual y la capacidad de consumo.

En el trabajo recordamos lo que fue la campaña continental de los quinientos años de resistencia indígena, negra y popular como correlato de la celebración oficial de los quinientos de lo que el discurso eurocéntrico ha llamado "el descubrimiento de América". Recordamos la década de los noventa, marcada por la insurrección zapatista en México, y los procesos iniciados en una buena parte de la región: Guatemala, Brasil, Ecuador, Bolivia, Colombia, Venezuela, gracias a las grandes movilizaciones y amplio despertar de movimientos campesinos sin tierra, indígenas y afrodescendientes, pobladorxs popularxs urbanxs, entre otros. Finalmente, se reconoce la presencia y el acompañamiento de una parte del feminismo a estos procesos. 
Sin embargo, desarrollando una mirada no idealizadora, advertimos las dificultades y obstáculos de los cuales ha estado plagado el camino de estos intentos de articulación entre feminismo y luchas indígenas, negras y populares.

Aunque observamos los intentos de encuentro y reconocimiento mutuo entre las feministas y las "mujeres" de los movimientos indígenas y afros, al mismo tiempo se han hecho más que evidentes los problemas y peligros del procurar la construcción de una agenda de intereses comunes. La historia demuestra la imposibilidad de una escucha atenta más equitativa y horizontal; la imposibilidad para el feminismo de abandonar su pretensión de producir una verdad universal sobre la opresión basada en género y los caminos para revertirla.

Así, no siempre resultaron fáciles las articulaciones, complicidades y alianzas entre mujeres de los movimientos indígenas, afrodescendientes y populares con las feministas. Ello se debió, entre otras cuestiones, al origen de clase y raza de las feministas, ya que, si bien había mujeres descendientes de pueblos originarios y africanos, provenientes de la clase trabajadora, en sus filas, lo cierto es que la gran mayoría de las feministas han sido blanco-mestizas, urbanas, universitarias, provenientes de clases medias y altas. Tal como lo han denunciado y analizado las feministas negras y del movimiento de color en los Estados Unidos (Hooks, 2004; Lorde, 2003), este origen condicionó las interpretaciones de la opresión de las mujeres, así como los postulados básicos del programa liberador a desarrollar, es decir, las estrategias que permitirían acabar con esta opresión y el tipo de sociedad al que aspiramos. Con ello, las diferencias entre las feministas y las mujeres organizadas de grupos subalternos no se hicieron esperar. Estas últimas no se han sentido atraídas ni convocadas por la lucha feminista, una lucha que han visto bastante alejada de su realidad (Espinosa, Gómez y Ochoa, 2014, p. 22).

A pesar de que cada vez más voces de "mujeres" indígenas y afrodescendientes son capaces de reconocer, observar y visibilizar el sexismo que opera en sus comunidades y en sus organizaciones políticas, sigue existiendo una frontera y una relación complicada entre el feminismo y las "mujeres" de las organizaciones o de las comunidades y movimientos indígenas y afro. 
A mi entender, ello tiene que ver con algunas otras razones que me gustaría retomar al finalizar estas reflexiones.

Lo cierto es que con la expansión del feminismo a espacios más amplios de la vida en sociedad y la incorporación de compañeras racializadas y de comunidades marginales, estos problemas ya no solo se expresaron en una relación entre movimientos, sino en las mismas filas feministas. Los desacuerdos se manifestaron en la experiencia de unas relaciones desiguales “entre mujeres". Experimentar en el activismo feminista el eurocentrismo, la violencia simbólica y epistémica, el racismo, la meritocracia y otras formas de manejo y traspaso de los lugares de prestigio y de poder, de manejo de la palabra y la representación, ha sido para muchas de nosotras la herida que nos marcó e impulsó a la búsqueda de explicaciones que nos permitieran comprender y dar cuenta de lo vivido. Una experiencia de la opresión que el feminismo en su forma clásica, en sus verdades compartidas, negaba sistemáticamente y no nos permitía ver y analizar en su justa dimensión.

Y es desde ahí que varias de nosotras en Abya Yala hemos denunciado y tematizado estos problemas en las organizaciones feministas y el movimiento más amplio. Las primeras denuncias acerca de los privilegios y prerrogativas que gozaban algunas mujeres, y que se reflejaban en la organización misma en términos de definición de problemáticas centrales, estrategias, alianzas y formas de representación, fueron formuladas en clave de clase desde mediados de la década de los ochenta por el feminismo popular latinoamericano, comprometido con el marxismo y la política de izquierda. Posteriormente, durante la década de los noventa, estas denuncias serían formuladas por el feminismo autónomo en términos de la relación con el Estado y los procesos de institucionalización y burocratización de la agenda feminista.

Pero, paralelamente a esta historia, desde los ochenta apareció en la escena pública la fuerza de un movimiento negro en Brasil, de donde emergerían las primeras voces de mujeres negras dispuestas a pelear su lugar tanto en el movimiento antirracista mixto como en el feminista. El movimiento afrobrasileño será paradigmático en esta historia, inaugurando una conciencia de raza y una lucha amplia contra el racismo institucional. El feminismo afrobrasileño sería pionero en la región, en la apertura a pensar la 
relación entre género, raza y clase ${ }^{2}$. En este país, de mayoría afrodescendiente, el llamado movimiento de mujeres negras se nutrió de las producciones minoritarias de las feministas negras en los EE.UU. y, en diálogo con ellas, ha podido avanzar desde la década de los ochenta algunas herramientas teórico-metodológicas para pensar su propia realidad ${ }^{3}$.

\section{De las dificultades de producir una conciencia decolonial y antirracista y las influencias del feminismo negro y de color en los EE.UU. en Abya Yala}

Es imposible negar la gran influencia que el feminismo negro y de color de EE.UU. ha tenido sobre las feministas antirracistas en América Latina y en otras latitudes. Ello se debe seguramente a lo que, acogiéndome al análisis decolonial, nombro como una geopolítica del conocimiento (Walsh, 2002) o, aún mejor, una economía política del conocimiento (Rivera Cusicanqui, 2010). La preocupación respecto de la imposibilidad histórica del feminismo latinoamericano, de producir un teorización propia que atienda a su misma configuración geopolítica, ha sido expresada ya por autoras como Breny Mendoza (2009) y Mayra Leciñana (2003) y en mi propia obra fue lo que motivó a que llevara a cabo, junto a un grupo de estudiantes y activistas, la investigación independiente sobre la producción de conocimientos por parte de los Estudios de Género y Sexualidad en América Latina (Espinoza y Castelli, 2011).

La condición de países satélite del colonialismo europeo y, posteriormente, norteamericano nos define como países receptores de conocimiento y no productores. Esto ha permitido que el pensamiento feminista negro y de color, a pesar de la condición de subalternidad que ha tenido en la academia de los Estados Unidos, lograra cierto nivel de recepción y se convirtiera en

2 En el III Encuentro Feminista de América Latina y el Caribe, celebrado en Brasil en 1985, se desató una situación límite que refiere a esta tensión. Un grupo de mujeres negras y pobres, provenientes de las favelas de Río de Janeiro, intenta entrar al encuentro gratuitamente. Si bien la comisión organizadora señaló el gran número de becas que habían sido otorgadas para mujeres negras y pobres, y denunció la maniobra política de los partidos políticos para desacreditar al feminismo, el incidente fue motivo para que "muitas das participantes, especialmente militantes do então emergente movimento de mulheres negras, insistiram que as questões de raça e classe não ocupavam um lugar central na agenda do Encontro e que as mulheres negras e pobres não haviam tido uma participação significativa na elaboração dessa agenda" (Álvarez, et. al., 2003, p. 548).

3 No podemos dejar de hacer referencia aquí a las producciones de teóricas-activistas negras como Luiza Bairros, Leila Gonzáles, Suely Carneiro, Jurema Wernerk, entre otras. 
una referencia de la voz de las mujeres racializadas y del tercer mundo. Tal ha sido la importancia de este pensamiento para reflexionar sobre la relación entre raza y género en América Latina, que hemos tenido que enfrentarnos al inconveniente de que sus representantes terminen suplantando las voces locales que intentamos aportar en esta misma línea de argumentación. Así, se da continuidad a la larga tradición que desconoce, sistemáticamente, los aportes locales, obstaculizando el desarrollo de una teoría propia que parte de nuestra impronta constitutiva. Este problema se advierte incluso en un campo de investigación como el giro decolonial, que ha denunciado la colonialidad del saber y que, sin embargo, a la hora de acercarse a pensar la relación entre colonialidad y clasificación de género acude a las interpretaciones desarrolladas por voces provenientes del feminismo negro, chicano y de color en los EE.UU., dando por hecho que ellas representan las voces de las subalternas latinoamericanas y caribeñas (Mendoza, 2014).

Más allá de la deuda histórica con el reconocimiento, la valoración y el desarrollo de una teoría propia, considero que la influencia decisiva que ha tenido el feminismo negro y de color de los EE.UU. en el desarrollo de apuestas antirracistas en nuestra región se debe a ciertas condiciones de posibilidad, que propiciaron tempranamente la aparición histórica de este pensamiento en los EE.UU., mucho antes que en América Latina.

Propongo pensar que algunas de las condiciones de posibilidad para el surgimiento cronológicamente diferenciado de los activismos y teorías feministas antirracistas en EE.UU. y en América Latina podrían tener que ver con lo que Antonio Guimarães (1996) explica como la conformación histórica de diferentes modelos de racismo estatales en el nivel mundial. Siguiendo la hipótesis de Guimarães, la existencia de un modelo de segregación racial como el estadounidense permitiría el surgimiento temprano -en la temporalidad marcada por la configuración de los Estados nación y la colonialidad- de una conciencia de "opresión racial" en EE.UU., distinto a lo que ocurriría en países de América Latina, en donde un tipo de racismo "asimilacionista", derivado de la estrategia y la ideología del mestizaje ${ }^{4}$,

4 Existe una amplia obra que analiza el mestizaje como discurso de las élites criollas en América Latina. Para algunos análisis interesantes sobre el mestizaje como ideología ver, entre otrxs: Breny Mendoza (2001), Mary Louise Pratt (1997), Marisol de la Cadena (2007), Peter Wade (1997). 
impediría o retardaría la aparición de una conciencia de opresión racial y una política derivada de ella.

La ideología del mestizaje ha instalado la idea de la posibilidad de dirimir los conflictos entre diferentes tradiciones culturales y epistémicas enfrentadas, donde se imponía el abandono de las epistemologías autóctonas y su sustitución por la matriz moderna colonial, a través de los Estados nación latinoamericanos. Por medio de un discurso que oculta más de lo que muestra, "plagado de eufemismos que velan la realidad en lugar de designarla” (Rivera, 2010, p. 19), las élites nacionales ofrecían a las poblaciones no blancas un discurso mistificador y de integración, al tiempo que desarrollaban dispositivos de blanqueamiento amplio, los cuales, en su concepción, permitirían que llegáramos a ser el tipo de nación adelantada y desarrollada que debíamos llegar a ser, a la manera de Europa. Este proceso de blanqueamiento ha sido sustantivo en la conformación tanto de las clases dominantes como de las clases medias y trabajadoras urbanas, formadas bajo los ideales de la modernidad. Las comunidades en resistencia fueron sistemáticamente sometidas a exterminio y exclusión o, en caso contrario, obligadas a olvidar su origen y adscribirse al ideal moderno occidental mediante la propuesta del mestizaje integrador (Mendoza, 2001).

Habría que decir que el origen mayoritariamente burgués y blanco/ mestizo del feminismo ha significado en Latinoamérica una adscripción, pero también un compromiso, a estos ideales emancipatorios de progreso, igualdad, libertad tanto personal como sexual (Espinosa, 2010 y 2015). Ello ha implicado la producción de una mirada euro centrada, que no puede observar los efectos del racismo como episteme sobre la que se funda el propio programa liberatorio latinoamericano y las formas contemporáneas de nuestra organización política y social. Y así, al feminismo en América Latina le ha sido difícil y le sigue costando admitir su complicidad con la expansión de la mirada moderna colonial, el racismo y el sistema de género racializado que de allí se deriva.

Esta particularidad histórica nos ha diferenciado de lo que ha ocurrido en otros contextos geopolíticos como África y EE.UU., donde fue evidente el racismo constitutivo gracias a experiencias de segregación y apartheid (Guimarães, 1996). Así, un primer movimiento contemporáneo de feministas antirracistas en EE.UU. tuvo posibilidades de surgir, desde principios 
de los años setenta del siglo pasado, gracias a la conjugación de dos fuertes movimientos que aparecen simultáneamente en esta década: el feminista y el de los derechos civiles, posteriormente radicalizado en movimientos nacionalistas negros, muchos de ellos adhiriendo al análisis marxista. Es desde la experiencia de activismo en estos dos movimientos y de la militancia marxista que surgirán las voces de las feministas negras y de color en EE.UU.

El giro que inaugura este feminismo subalterno de "mujeres" racializadas, provenientes de la clase trabajadora en EE.UU., solo fue posible gracias a que ellas logran conceptualizar e introducir la categoría de raza como histórica, que viene a jugar un papel crucial en la acumulación y expansión capitalista, y que permite comprender la opresión que sufren una buena parte de las "mujeres", opresión de la que la teoría feminista, euro centrada, no ha podido dar cuenta.

Las feministas negras y de color, nutridas por la experiencia del separatismo, el nacionalismo y la militancia revolucionaria negra y chicana, bebieron de una teoría marxista radical y revisitada que pudo relacionar clase y raza de manera efectiva. Esta relación permitió reparar en el sujeto subalterno, producido por la expansión del capital a través de la empresa colonizadora, un sujeto racializado a efectos de justificar la superioridad blanca y que el pensamiento marxista no pudo teorizar sino limitadamente, gracias a su fuerte compromiso con el programa moderno ilustrado.

Este sujeto político, producido desde una conciencia de raza, debatirá el eurocentrismo epistémico y el proyecto colonialista expansivo de Europa, denunciado ya por autores claves del movimiento de la negritud, como Frantz Fanon (1965a y 1965b) y Aimé Cesaire (2006), entre otros, desde mediados de la década de 1930 del siglo pasado. A partir, de allí fue posible comenzar a pensar una "diferencia" con el sujeto europeo de la emancipación y con el programa político del socialismo internacional. Esta diferencia o especificidad comenzará a ser tematizada por las feministas negras, quienes llevarán a cabo una labor de revisión de las premisas básicas que explican el sometimiento de las mujeres en el patriarcado; premisas que habían sido formuladas y sostenidas por el feminismo blanco burgués, incluso el comprometido con la lucha de clases.

Por el contrario, en el feminismo latinoamericano hemos necesitado más tiempo para que aparezcan voces de mujeres y feministas racializadas 
conscientes de la opresión racista y sexista. Y mucho más se ha necesitado para que el feminismo latinoamericano, en su conjunto, se haga consciente de la necesidad de articular la preocupación por el racismo.

Aunque a principios de los años noventa asistimos al nacimiento de un movimiento latinoamericano de mujeres negras liderado por feministas negras ${ }^{5}$, por cierto, y no es un dato menor, varias de ellas lesbianas, ello ha sido a contrapelo de los intereses y la sensibilidad del mainstream feminista local. Como he señalado en un trabajo anterior (Espinosa Miñoso, 2012), es ilustrativo el hecho de que, a pesar del consumo permanente en América Latina de la teorización feminista producida en EE.UU. y Europa, la producción crítica desarrollada por el movimiento de feministas tercermundistas en EE.UU., así como las primeras incursiones locales dirigidas en este sentido, no fueron objeto, durante largo tiempo, de una particular atención por parte del feminismo latinoamericano en su conjunto. Como advierto, "hubo que esperar a que estos aportes fueran recogidos y valorados por las académicas blancas norteamericanas para que gozaran de algún nivel de [mínima atención y] legitimidad en Latinoamérica" (Espinoza, 2012, p. 217). Esta forma de recepción hace marginal, y sobre todo problemáticos, sus usos e impactos en la teorización feminista contemporánea tanto internacional como latinoamericana.

De todas formas, las feministas negras y de color en EE.UU. han sido los grandes referentes teórico-políticos de nosotras, las feministas antirracistas en América Latina. Sus postulados y críticas han sido esenciales para ayudar a configurar una voz propia desde posiciones subalternas del género. Esta voz en plena producción, por cierto, sin desmeritar la genealogía de la que se ha nutrido, debe, sin embargo, continuar su propio camino aportando desde la experiencia de la colonialidad de poder, del ser y del saber a este andamiaje crítico proveniente de las racializadas subalternas de este mundo.

La experiencia de la colonialidad no es algo que las feministas antirracistas norteamericanas hayan vivido o teorizado, aun a pesar de que ellas han estado atentas al colonialismo y el imperialismo que conocen debido a la historia de esclavitud y del colonialismo interno, así como a la experiencia

5 Durante este periodo, han surgido diferentes organizaciones de mujeres negras en la región -a manera de ejemplo: La Casa de la Mujer Afro en Rep. Dominicana y, en el nivel continental, la Red Latinoamericana de Mujeres Afro (Curiel, 2007). 
de la migración que muchas han experimentado como latinas en EE.UU. Las feministas antirracistas en Abya Yala tienen entonces mucho para aportar a un marco que interprete, eficazmente, la relación entre la opresión/ dominación de las mujeres y el racismo. Este marco que, desde mi punto de vista, es el del análisis de la colonialidad y del sistema moderno colonial de género permite profundizar y mejorar la crítica producida por las feministas antirracistas en EE.UU., y la primera camada de feministas antirracistas en Abya Yala a la vez, da nuevas pistas para superar los obstáculos epistemológicos que contiene la teoría de la interseccionalidad, que, como sabemos, es considerada el aporte fundamental de los llamados feminismos negros.

\section{Sobre los límites de una teoría centrada en la opresión de género y sus implicaciones negativas en una lucha unificada}

La aparición de una conciencia de género es bastante nueva en la historia de los movimientos sociales amplios en América Latina. Hemos sido testigos de cómo, con el pasar del tiempo, el discurso feminista en América Latina ha logrado impactar en el nivel de las ideas en determinados espacios de los movimientos anticolonialistas, anticapitalistas, populares y étnico-raciales amplios. Esto se puede observar en algunos análisis que de allí se originan, y con los cuales se evidencia el nacimiento de una preocupación cada vez mayor por la opresión sexista. Es el resultado del discurso estatal sobre derechos, que se ha ido logrando con dificultad gracias a las presiones del movimiento feminista y el trabajo sistemático del feminismo en el Estado, las ONG y la agenda del desarrollo. También tiene que ver con años de trabajo e inserción en las comunidades del llamado feminismo popular y de izquierda. Asimismo, parte de los feminismos autónomos y radicales en América Latina ha sido proclive a ver la necesidad de mantener y comprometerse con lo que han considerado como "otras luchas" que se llevan a cabo en el continente. Finalmente, no hay que escatimar las influencias de la academia feminista, en la medida de su expansión en las universidades latinoamericanas, y la aparición de programas de acción positiva por medio de los cuales mujeres de origen indígena y afro han podido acceder a la profesionalización en los estudios de género y sexualidad. Todo ello ha contribuido a la expansión de las ideas feministas y cierto nivel de 
popularización de sus nociones e interpretaciones sobre la opresión de género (o de las mujeres por ser mujeres).

Gracias a ello, los movimientos amplios anticoloniales, antiimperialistas y antirracistas de nuestro continente empiezan a incorporar con timidez, pero mucho más rápido de lo que se está en disposición de admitir, una preocupación por reproducir relaciones jerárquicas entre mujeres y varones, y entre géneros y sexualidades despreciadas, así como entre aquellas que son normativas al interior de las comunidades al igual que de los propios movimientos.

Pero habría que sospechar del tipo de verdad sobre el "género" que el feminismo latinoamericano sigue instalando en su expansión, el tipo de interpretación histórica que hace para intentar explicar lo que define como "una opresión común de las mujeres por el hecho se ser mujeres". A pesar de que desde distintos frentes de la teorización feminista se ha intentado mostrar los profundos problemas de una conceptualización universalista y fragmentada de la opresión, la razón feminista ${ }^{6}$ en América Latina en su avance persiste en proponer un análisis homogeneizador.

Los límites de esta teorización se expresan cotidianamente en una política feminista latinoamericana cuyas estrategias, focalizadas alrededor del género, o lo que otras nombran como "la condición femenina", pretenden impactar al conjunto de las mujeres por sobre las condiciones sociales, culturales y económicas de los grupos y comunidades a los que ellas pertenecen. Como he señalado en otras ocasiones (Espinosa, 2014 y 2016), este supuesto resulta productivo a las mujeres que gozan de privilegios de clase y raza, en tanto se benefician de una política que deja inamovibles aquellos

6 Aquí es importante el concepto de "razón” y el cómo lo estoy entendiendo. Sostengo que hay una razón feminista universal, que consiste en una serie de principios sobre los que nos hemos asentado las feministas de todos los tiempos y de las más diversas corrientes contemporáneas, tanto en Estados Unidos como en Europa y en América Latina, Asia o África. Esta razón se ha caracterizado por su compromiso con la modernidad y, por consiguiente, con su cara oculta: la colonialidad. Acogiéndome a la caracterización desarrollada por Mario Blaser, digo que hay al menos tres cuestiones que son sustantivas al mito moderno y que el programa feminista reproduce: "la gran separación entre naturaleza y cultura, la diferencia colonial entre modernos y no-modernos, y una temporalidad lineal unidireccional que discurre desde el pasado hacia el futuro" (Blaser, 2013, p. 24). Estas ideas las desarrollo con mayor minuciosidad en un trabajo de próxima aparición (Espinosa Miñoso, Hacer genealogía de la experiencia: el método hacia una crítica a la colonialidad de la Razón feminista desde la experiencia histórica en América Latina, 2017). 
ámbitos de la vida social, en donde ocupan lugares de jerarquía, como parte de aquellos grupos dominantes que han ejercido el poder históricamente?

Esto tiene consecuencias directas en el tipo de política feminista que se lleva a cabo y en el de valoración que hacen las "mujeres" racializadas de las comunidades marginales urbanas y campesinas. Cuando estas se ven compelidas a superponer una alianza de género por sobre una alianza de clase y raza, no dudan en decidir a cuál dar prioridad. Saben que en la alianza de género que les propone el feminismo tienen las de perder, puesto que el costo que se les exige es estar dispuestas a abandonar, o relegar a segundo plano, los antagonismos históricos que les condenan como parte de una comunidad o tipo de pueblo. Intuyen o parten de la experiencia para saber que una vez que se acabe la marcha, la reunión o el encuentro de mujeres; que se alcancen los que se anuncian como "objetivos comunes", ellas volverán solas a vérselas con la dura realidad de una vida condenada a formas históricas de violencia institucional y estatal, condiciones a las que en lo cotidiano se enfrentan junto a aquellos que precisamente el feminismo les presenta como "el enemigo interno" por combatir.

Esta conciencia de resistencia y sobrevivencia como pueblo, comunidad, grupo étnico-racial o clase es la que interviene a la hora de sopesar los pros y los contras de nombrarse o no feministas. Es la razón oportuna por la que con o sin una gran teoría que las respalde saben que el feminismo no es su lugar, que la propuesta feminista puede aportarles algunas cuestiones para su propia resistencia y su propia liberación, pero que esta no es su lucha. "No me salvo sola", me dijo una vez Julia Ramos, una dirigente aymara de la Confederación Bartolina Sisa de Bolivia ${ }^{8}$, para expresar su decisión de no nombrarse feminista: no se trataba solo de un nombre, sino de los objetivos de una lucha.

Mientras para el ideario feminista consensuado la lucha es una centrada en género y se hace "entre mujeres", las mujeres racializadas y los feminismos que nacen de esta experiencia piensan y hacen el esfuerzo de teorizar la

7 Es lo que he denominado como el racismo de la categoría de género cuando se convierte en la categoría primordial del análisis feminista: "Una imposibilidad de la teoría feminista de reconocer su lugar de enunciación privilegiado dentro de la matriz moderno colonial de género, imposibilidad que se desprende de su negación a cuestionar y abandonar este lugar a costa de "sacrificar", invisibilizando diligentemente, el punto de vista de "las mujeres" en menor escala de privilegio, es decir las racializadas empobrecidas dentro de un orden heterosexual" (Espinoza, 2016, p. 50).

8 Entrevista realizada por Yuderkys Espinosa, La Paz, Bolivia, 2010. 
opresión de un modo complejo, multidimensional y no fragmentado; para nosotras es fundamental la necesidad de una lucha común con los varones de la comunidad, pues sabemos que tanto como nosotras son cuerpos producidos por la matriz de opresión, disponibles para la explotación y la violencia.

Esta posición sigue sin poder ser digerida por la gran mayoría de las corrientes feministas latinoamericanas producidas en la colonialidad y la mirada eurocentrada. En la medida en que se afianza y profundiza un pensamiento antirracista y decolonial en el continente, nos topamos con resistencias fuertes al abandono de la centralidad y productividad del género como categoría dominante para explicar la opresión. El feminismo latinoamericano hoy puede estar más dispuesto a poner atención al racismo y los efectos del colonialismo. Pero su mirada del racismo sigue siendo superficial, particularista y, sobre todo, fragmentada y sumativa. Su comprensión y tratamiento del racismo y del sistema mundo-moderno-colonial-capitalista mantiene las categorías dominantes de opresión como si fueran de distinta índole y matriz histórica. Al final, hasta el mejor intencionado feminismo sigue pensando estas cuestiones como problemas separados, anexos a la dominación de género y, por tanto, menos sustantivos a las luchas de las mujeres.

Mientras no sea posible desandar este tratamiento de la opresión, el feminismo latinoamericano estará condenado a ser la lucha de unas pocas, pero aún más importante, a ser la lucha para la emancipación de unas y la ampliación de la opresión de las personas racializadas.

\section{Referencias}

Alvarez, S., Friedman, E., Beckman, E., Blackwell, M., Stoltz, N., Lebon, N., Navarro, M. y Ríos, M. (Julho-dezembro, 2003). Encontrando os feminismos latino-americanos e caribenhos. Estudos Feministas, 11(2), 541-575.

Bairros, L. (1995). Nossos Feminismos Revisitado. Revista Estudos Feministas, 3, 458-463. Blaser, M. (2013). Un relato de globalización desde el Chaco. Popayán: Universidad del Cauca. Césaire, A. (2006). Discurso sobre el colonialismo. Madrid: Akal.

Curiel, O. (2007). La Red de Mujeres Afrolatinoamericanas y Afrocaribeñas: un intento de acción política transnacional atacado por la institucionalización. En F. Pisani, N. Saltalamacchia, A. Tickner, N. Barnes, F. Pisani \& I. T. ITAM (Edits.), Redes transnacionales en la Cuenca de los Huracanes. Un aporte a los estudios interamericanos (253-279). México, D.F.: Cámara de Diputados, LX Legislatura, Estados Unidos Mexicanos. 
De la Cadena, M. (2007). ¿Son los mestizos híbridos? Las políticas conceptuales de las identidades andinas. En M. de la Cadena (Ed.), Formaciones de indianidad.Articulaciones raciales, mestizaje y nación en América Latina (p. 83-116). Colombia: Envión.

Espinosa, Y. (2010). Etnocentrismo y colonialidad en los feminismos latinoamericanos: Complicidades y consolidación de las hegemonías feministas en el espacio transnacional. Revista Venezolana de la Mujer, 14(33), 37-54.

Espinosa, Y. (2012). Los desafíos de las prácticas teórico-políticas del feminismo latinoamericano en el contexto actual. En M. Daza, R. Hoetmer \& V. Vargas (Edits.), Crisis y movimientos sociales en nuestra América. Cuerpos, territorios e imaginarios en disputa (209-226). Lima: Programa Democracia y Transformación Global (PDTG), Colección: Teorías Críticas y Transformación Global.

Espinosa, Y. (marzo-abril de 2014). Una crítica descolonial a la epistemología feminista crítica. El Cotidiano, 29(184), 7-12.

Espinosa, Y. (2015). El futuro ya fue: una crítica a la idea del progreso en las narrativas de liberación sexo-genéricas y queer identitarias en Abya Yala. En R. Moarquech Ferrera-Balanquet (comp.), Andar erótico decolonial (21-39). Buenos Aires: Ediciones El Signo, colección El desprendimiento.

Espinosa, Y. (2016). Y la una no se mueve sin la otra: descolonialidad, antiracismo y feminismo. Una trieja inseparable para los procesos de cambio. Revista Venezolana de Estudios de la Mujer, 21(46), 47-64.

Espinosa, Y. (2017). Hacer genealogía de la experiencia: el método hacia una crítica a la colonialidad de la Razón feminista desde la experiencia histórica en América Latina. En K. Ochoa, M. T. Garzón, Y. Espinosa Miñoso, A. Cumes \& B. Mendoza (Edits.), (An) danzas de los feminismos descoloniales y anti-coloniales en Abya Yala. Madrid: Akal.

Espinosa, Y. \& Castelli, R. (2011). Colonialidad y dependencia en los estudios de género y sexualidad en América Latina: el caso de Argentina, Brasil, Uruguay y Chile. En K. Bidaseca \& V. Vázquez Laba (Comp.), Feminismos y Poscolonialidad. Descolonizando el feminismo desde y en América latina (191-214). Buenos Aires: Godot.

Espinosa, Y., Gomez Correal, D. \& Ochoa Muñoz, K. (Edits.). (2014). Tejiendo de Otro Modo: Feminismo, epistemología y apuestas descoloniales en Abya Yala. Popayán: Editorial de la Universidad del Cauca.

Fanon, F. (1965). Los condenados de la tierra. México: Fondo de Cultura Económica.

Fanon, F. (1965b). Por la revolución africana: Escritos políticos. México, D.F.: Fondo de Cultura Económica.

Guimaräes, A. S. (Julio-agosto, 1996). El mito del anti-racismo en Brasil. Nueva Sociedad, $144,32-45$.

Leciñana, M. (Diciembre, 2003). Feminismo filosófico en el contexto latinoamericano: ¿quién habla y cómo? Subjetivación política y subalternidad. XII Congreso Nacional de Filosofía. Neuquén: Universidad Nacional del Comahue.

Mendoza, B. (2001). La desmitologización del mestizaje en Honduras. Evaluando nuevos aportes. Mesoamérica, 22(42), 256-279.

Mendoza, B. (2009). Los feminismos y la otra transición a la democracia de América Latina. En M. A. García de León (Ed.), Rebeldes Ilustradas. Barcelona: Libros de Revista Anthropos. 
Mendoza, B. (2014). La epistemología del sur, la colonialidad del género y el feminismo latinoamericano. En Y. Espinosa Miñoso, D. Gómez Correal \& K. Ochoa Muñoz (Edits.), Tejiendo de Otro Modo: Feminismo, epistemología y apuestas descoloniales en Abya Yala (91-104). Popayán: Editorial de la Universidad del Cauca.

Mohanty, C. T. (2008). Bajo los ojos de Occidente. Academia feminista y discurso colonial. En R. A. (Coord.), Descolonizar el Feminismo: Teorías y Prácticas desde los Márgenes (p. 117-164). Madrid: Cátedra.

Moraga, C. y Castillo, A. (1979). Esta puente mi espalda. Voces de mujeres tercermundistas en los Estados Unidos. San Francisco: Ism Press.

Pratt, M. L. (1997). Ojos Imperiales. Literatura de viaje y transculturación. Buenos Aires: Universidad Nacional de Quilmes.

Rivera Cusicanqui, S. (2010). Ch'ixinakax utxiwa: una reflexión sobre prácticas y discursos descolonizadores. Buenos Aires: Tinta Limón.

Spivak, G. C. (1998). ¿Puede hablar el sujeto subalterno? Orbis Tertius, III(6), 175-235.

Wade, P. (1997). Gente negra, nación mestiza. Dinámicas de las identidades raciales. Bogotá: Uniandes/Universidad de Antioquia/Siglo del Hombre.

Walsh, C. (2002). Las geopolíticas del conocimiento y colonialidad del poder. Entrevista a Walter Mignolo. En C. Walsh, F. Schiwy \& S. Castro-Gómez (Edits.), Indisciplinar las ciencias sociales: Geopolíticas del conocimiento y colonialidad del poder. Perspectivas desde lo andino (17-44). Quito: Universidad Andina Simón Bolivar, Ediciones Abya Yala. 